\title{
CONCLUSIONS
}

\section{Trends in regenerative therapies, combination approaches, and clinical highlights for spinal cord injury (SCl) regeneration}

\author{
Filippo Rossi, PhD ${ }^{1}$, Mario Gimona, PhD ${ }^{2}$, Giuseppe Perale, $\mathrm{PhD}^{3,4,5}$ \\ ${ }^{1}$ Department of Chemistry, Materials and Chemical Engineering "Giulio Natta", Milan, Italy; \\ ${ }^{2}$ GMP Laboratory, Spinal Cord Injury and Tissue Regeneration Center Salzburg (SCI-TReCS), \\ Paracelsus Medical University (PMU), Salzburg, Austria; ${ }^{3}$ Department of Neuroscience, IRCCS \\ Istituto di Ricerche Farmacologiche "Mario Negri”, Milan, Italy; ${ }^{4}$ Faculty of Biomedical Sciences \\ of the University of Southern Switzerland, Lugano, Switzerland; ${ }^{5}$ Ludwig Boltzmann Institute for \\ Experimental and Clinical Traumatology, Vienna, Austria
}

As anticipated in the forewords, this book aims at describing the current scenario in spinal cord injury (SCI) regeneration strategies, pointing toward most promising research directions in this wide and complex field. Recent results and clinical achievements from Prof. Courtine's team opened tremendous new directions for restoring patients' functionality, but not by directly regenerating injured tissue, rather by electronically bypassing the lesion gap.

Indeed, even if many preclinical studies and promising approaches have been proposed for SCI treatment basing on regenerative purposes, unfortunately nowadays none of them showed relevant efficacy when translated to human patients $[1,2]$. One of the main reasons is that many cell-based and/or pharmacological approaches are directed only to one specific and single pathway and did not consider combination-based therapies that simultaneously work on neuroprotection (against primary injury) and neuroregeneration of the damaged tissue [3]. Moreover, other problems are strictly related to conventional pharmacological treatments and potential side effects associated. Indeed the most commonly used strategy is represented by continuous infusion by minipumps [4,5]: advantages are associated with immediate drug efficacy and limited side effects. Unfortunately, various drawbacks restrict the applicability of this route of administration, such as limited drug diffusion into the spinal cord segment and cerebrospinal fluid clearance of about $5 \mathrm{~h}$. Higher doses and repeated injections are hence required. Furthermore, problems due to surgery and catheter placement are also frequently reported together with infections [4].

Recently, novel engineered biocompatible scaffolds obtained high interest in SCI treatment because they tackle some of the key features of this disease such as the 
localized trauma and the consequent presence of cyst that create a proper space for scaffold implantation or injection. Once in situ, such scaffolds can release drugs, cells, or biomolecules directly into the target area avoiding problems such as immune system clearance, systemic drug overdosage, or cells leakage into circulatory torrent.

In this field, medicine and engineering should work together to design suitable systems able to maintain local drug levels within a desired functional range, satisfying the need for fewer administrations and optimizing drug concentration and patient compliance. Promising results have been achieved with hydrogels, soft injectable polymeric matrices that can jellify in situ and then release multifunctional therapies directed to counteract multiple degenerative mechanisms of SCI, trying to release not only neuroprotective but also neuroregenerative agents as schematized in Figs. 15.1 and 15.2 [6].

Moreover, hydrogels can protect biomolecules reducing their deactivation and biodistribution of chondroitinase ABC and NT-3: respectively, a bacterial enzyme that is able to digest chondroitin sulfate glycosaminoglycans and a neurothrophine, both of which can promote axonal regeneration and sprouting supporting functional recovery in various preclinical models [7]. In general, if injected systemically, it is extremely difficult to retain them at the injury site because of their rapid diffusion into extracellular fluids: a single injection of neurotrophic factors has a limited half-life only of about $30 \mathrm{~min}$, and multiinjection rather than a single injection would hence be needed to provide a neuroprotective effect.

Following the same strategy, other factors, such as brain-derived neurotrophic factor [8], ciliary neurotrophic [9], epidermal [10], and fibroblast growth factors [11] are important promoting factors for neural regeneration and can hence be smartly delivered using hydrogels. Another interesting and very promising strategy
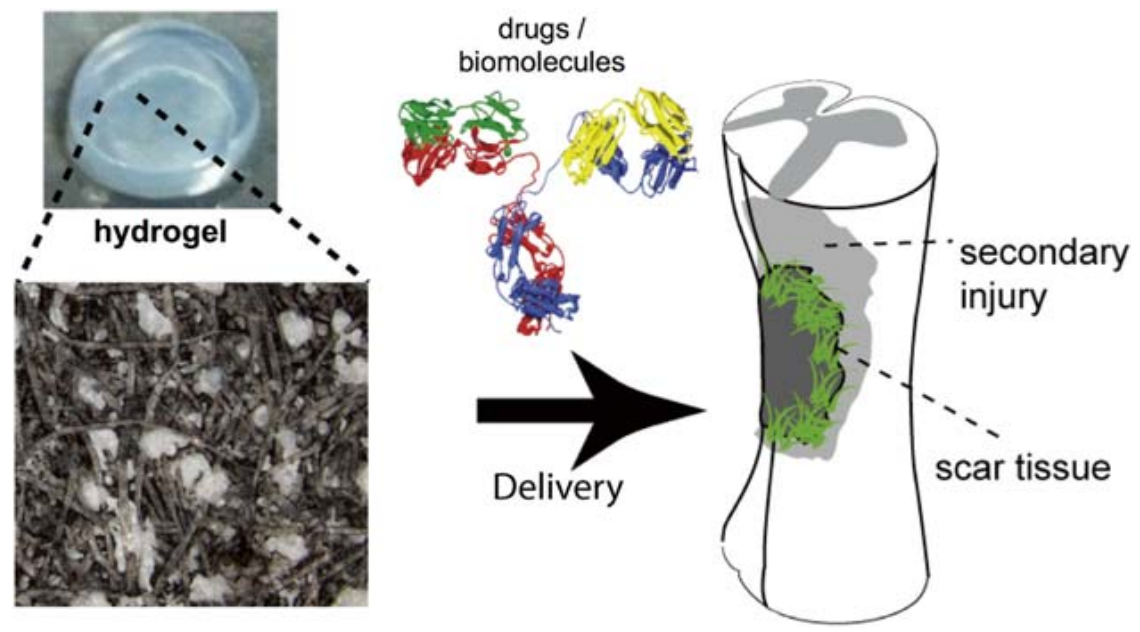

FIGURE 15.1

A cartoon represents delivery of drugs or biomolecules from the hydrogel in the damaged spinal cord. 

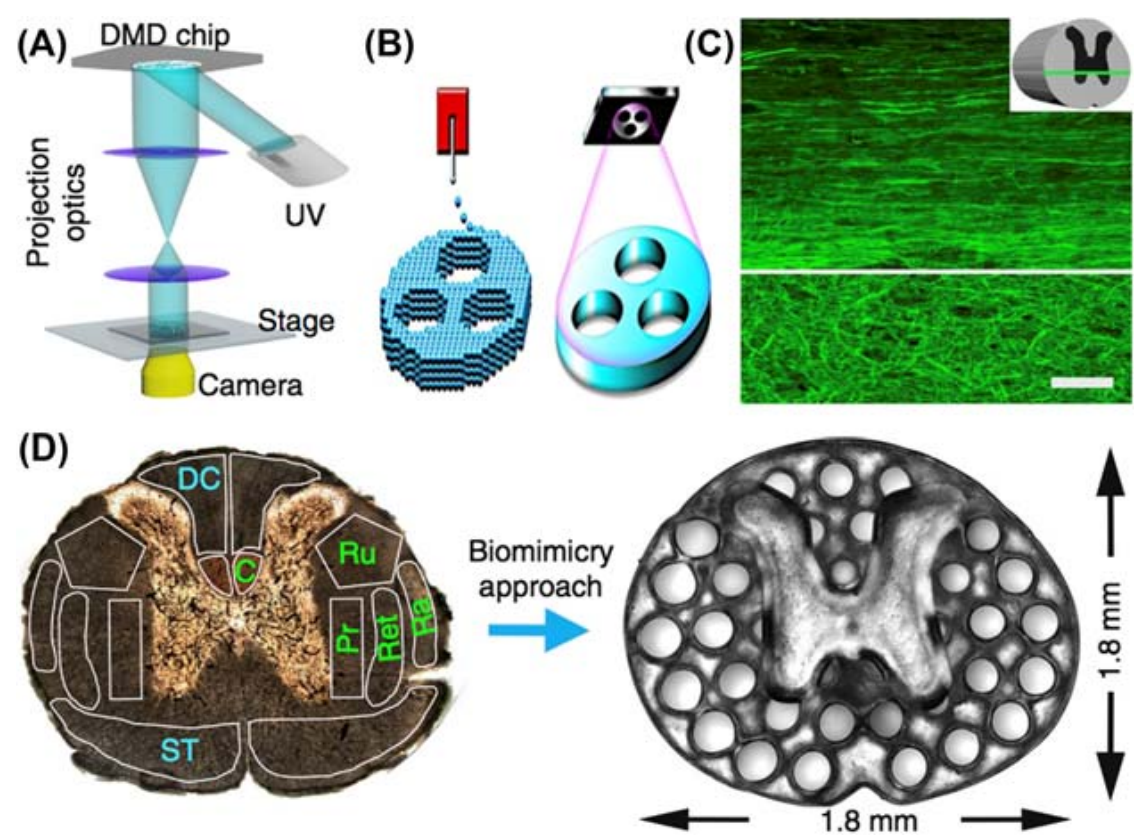

FIGURE 15.2

3D printer setup: An ultraviolet light source (365 nm wavelength); a computer for sliced image-flow generation and system synchronization;

a digital micromirror device (DMD) for optical pattern generation; a set of projection optics; a stage for sample position control; and a CCD (charge-coupled device) imaging system for online monitoring of the fabrication process.

Reprinted with permission from Koffler J, Zhu W, Qu X, Platoshyn O, Dulin JN, Brock J, et al. Biomimetic 3D-printed scaffolds for spinal cord injury repair. Nat Med 2019;25:263-269.

is represented by biofabrication of scaffolds able to mimic spinal cord tissue, as depicted in Fig. 15.2. In this direction, Koffler and coworkers used microscale continuous projection printing method to create the complexity of CNS structure [12].

In parallel, there has been growing interests in developing nanoscaled delivery tools able to enter within specific cells delivering selectively their contents, working so as Trojan horses $[13,14]$. Following this strategy, a new frontier is opened by smart combination of biomaterials to address multiple approach using both hydrogels and nanoparticles (NPs).

Naturally occurring nanoparticles, such as exosomes and other therapeutically active extracellular vesicles (EVs), are liberated by a plethora of cells, including mesenchymal stromal cells into their complex secretome, and hold great promise for future application in the acute to subacute phase of SCI. EV-enriched secretomes preserve and harness the immunomodulatory potential of mesenchymal-derived cells (MSCs) [15] and display neuroprotective activity in vivo and in vitro via multiple pathways. These allogeneic biological therapeutics present a much-improved 
safety profile compared with stem cells and, albeit little is known about the potential modes of action the impact on tissue mechanotransduction and compliance is evident. EV-rich allogeneic secretomes will also enable new approaches that consider the different steps of body reactions following traumatic SCI in a timedependent fashion and thus move SCI therapy into a 4D situation. The combination of regeneration-promoting (nanoporous) materials and structure-inducing scaffolds together with vesicular secretomes may be employed to augment the body's intrinsic abilities to repair damaged tissue and eventually prepare the required regenerative environment for functional recovery and subsequent therapeutic strategies, including cell-based approaches [16] (Fig. 15.3).

Indeed, the ability to remain localized in situ, together with the possibility of controlling the delivery of hydrophilic high steric hindrance molecules, typical of hydrogels, could be combined with the cell selectivity and with the possibility of tuning the release of hydrophobic drugs, typical role of nanoparticles. Several recent studies have investigated these aspects, trying to combine the advantages of both systems: minocycline [17], methylprednisolone [7], antibodies [18] and growth factors [19] were incorporated in NPs and then loaded into hydrogels to provide a sustained release into the final target tissue, aiming at increasing medical recovery chances. An ideal biomaterial-based delivery platform must achieve localized and sustained release and a favorable risk/benefit ratio to be adopted clinically for SCI treatment. In particular, all the following characteristics in developing delivery tools have to be considered:

1. biocompatibility;

2. controlled biodegradability;

3. easy injectability;

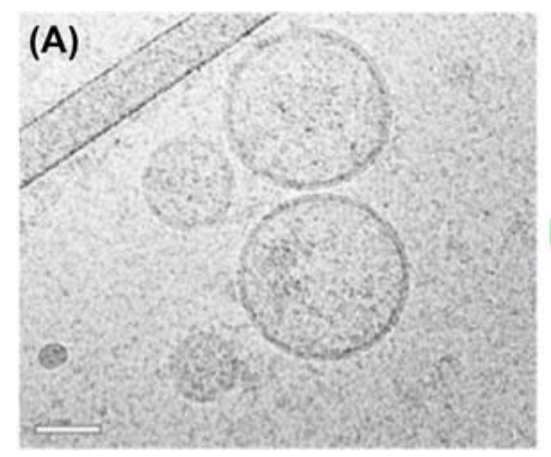

(B)

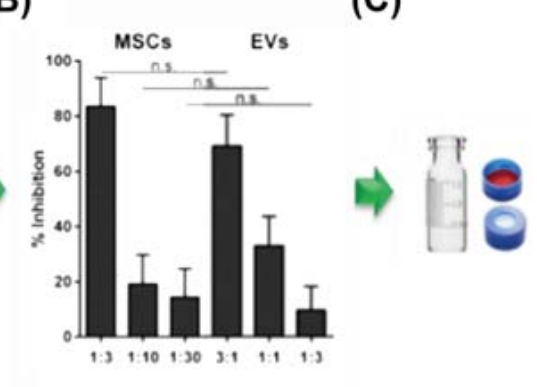

FIGURE 15.3

Mesenchymal-derived cell (MSC)-derived secretomes enriched in 50-130 nm sized membrane surrounded extracellular vesicles (EVs) (A) preserve the immunomodulatory function ( $B$; inhibition of T cell proliferation) of the parental cells and can be manufactured as ready-to-use injectable allogeneic therapeutics (C) for immediate application in the acute phase of spinal cord injury (SCI) (scale bar $=50 \mathrm{~nm}$ ). 
4. complete control over material formulation and hence complete rationalization in terms of physicochemical properties;

5. ability to sustain multiple drugs delivery, at the same time and with different and independent kinetic profiles;

6. total absence of any chemical interactions between moieties of materials and drugs.

These findings indicate that material science, in conjunction with bio- and nanotechnologies, can develop novel specifically designed devices able to maintain drug levels within a desired range, satisfying the need for fewer administrations, optimal use of the drug, and increased patient compliance.

Overall, the hope is that one of these therapies, or most likely a combination of some of them, will soon provide sufficiently robust animal data to withstand the regulatory and ethical hurdles to enter into clinical phase and in the end allow the achievement of effective results in patients. Even just partial recovery and preliminary clinical results will for sure open new directions in research that will finally lead to find a spectrum of solutions for this complex and high invalidating pathology.

\section{References}

[1] Morren JA, Galvez-Jimenez N. Current and prospective disease-modifying therapies for amyotrophic lateral sclerosis. Expert Opin Investig Drugs 2012;21:297-320.

[2] Rabchevsky AG, Patel SP, Springer JE. Pharmacological interventions for spinal cord injury: where do we stand? How might we step forward? Pharmacol Ther 2011;132: $15-29$.

[3] Kwon BK, Okon EB, Plunet W, Baptiste D, Fouad K, Hillyer J, et al. A systematic review of directly applied biologic therapies for acute spinal cord injury. J Neurotrauma 2011;28:1589-610.

[4] Belverud S, Mogilner A, Schulder M. Intrathecal pumps. Neurotherapeutics 2008;5: 114-22.

[5] Cohen SP, Dragovich A. Intrathecal analgesia. Anesthesiol Clin 2007;25:863-82.

[6] Perale G, Rossi F, Santoro M, Peviani M, Papa S, Llupi D, et al. Multiple drug delivery hydrogel system for spinal cord injury repair strategies. J Control Release 2012;159: 271-80.

[7] Lee H, McKeon RJ, Bellamkonda RV. Sustained delivery of thermostabilized chABC enhances axonal sprouting and functional recovery after spinal cord injury. Proc Natl Acad Sci USA 2010;107:3340-5.

[8] Liang W, Han Q, Jin W, Xiao Z, Huang J, Ni H, et al. The promotion of neurological recovery in the rat spinal cord crushed injury model by collagen-binding BDNF. Biomaterials 2010;31:8634-41.

[9] Burdick JA, Ward M, Liang E, Young MJ, Langer R. Stimulation of neurite outgrowth by neurotrophins delivered from degradable hydrogels. Biomaterials 2006;27:452-9.

[10] Hamann MCJ, Tator CH, Shoichet MS. Injectable intrathecal delivery system for localized administration of EGF and FGF-2 to the injured rat spinal cord. Exp Neurol 2005; 194:106-19. 


\section{CONCLUSIONS Trends in regenerative therapies}

[11] Vulic K, Shoichet MS. Tunable growth factor delivery from injectable hydrogels for tissue engineering. J Am Chem Soc 2012;134:882-5.

[12] Koffler J, Zhu W, Qu X, Platoshyn O, Dulin JN, Brock J, et al. Biomimetic 3D-printed scaffolds for spinal cord injury repair. Nat Med 2019;25:263-9.

[13] Papa S, Rossi F, Vismara I, Forloni G, Veglianese P. Nanovector-mediated drug delivery in spinal cord injury: a multitarget approach. ACS Chem Neurosci 2019;10:1173-82.

[14] Mauri E, Chincarini GMF, Rigamonti R, Magagnin L, Sacchetti A, Rossi F. Modulation of electrostatic interactions to improve controlled drug delivery from nanogels. Mater Sci Eng C 2017;72:308-15.

[15] Pachler K, Ketterl N, Desgeorges A, Dunai ZA, Laner-Plamberger S, Streif D, et al. An in vitro potency assay for monitoring the immunomodulatory potential of stromal cellderived extracellular vesicles. Int J Mol Sci 2017;18:E1413.

[16] Gomes ED, Silva NA, Salgado AJ. Combinatorial therapies for spinal cord injury: strategies to induce regeneration. Neural Regen Res 2019;14:69-71.

[17] Papa S, Rossi F, Ferrari R, Mariani A, De Paola M, Caron I, et al. Selective nanovector mediated treatment of activated proinflammatory microglia/macrophages in spinal cord injury. ACS Nano 2013;7:9881-95.

[18] Stanwick JC, Baumann MD, Shoichet MS. In vitro sustained release of bioactive anti-NogoA, a molecule in clinical development for treatment of spinal cord injury. Int J Pharm 2012;426:284-90.

[19] Piotrowicz A, Shoichet MS. Nerve guidance channels as drug delivery vehicles. Biomaterials 2006;27:2018-27. 